\title{
Grundsätze und Auswirkungen der Sparpolitik in Deutschland
}

\section{LILIANNA JODKOWSKA}

prof. dr, HTW Berlin,

e-mail: jodkows@htw-berlin.de

\section{Zasady i skutki polityki ostrego oszczędzania w Niemczech}

SŁoWA KLUCzOWE polityka ostrego oszczędzania, zadłużenie

StreszCZenie Polityka oszczędnościowa jest w Niemczech nadal bardzo popularna, chociaż analizy i porównania wykazują, że może mieć trwały, negatywny wpływ na wzrost gospodarczy i poziom inwestycji.

Cel - wyjaśnienie pojęcia ostrego oszczędzania, analiza wpływu wdrożenia polityki oszczędnościowej w Niemczech na tle innych krajów, analiza obecnego stanu wdrożenia polityki oszczędnościowej w Niemczech.

Metodologia - Podstawą badania jest analiza porównawcza wybranych badań i analiz wpływu polityki ostrego oszczędzania na wzrost gospodarczy oraz obliczeniach własnych korelacji zachodzącej pomiędzy wzrostem gospodarczym a zadłużeniem.

Wynik - Wyniki analiz są ambiwalentne. Istnieje wielu zwolenników i przeciwników polityki ostrego oszczędzania. Dotychczasowe analizy, poparte interpretacją danych makroekonomicznych i finansowych, potwierdzają zarówno pozytywne, jak i negytywne skutki polityki ostrego oszczędzania. Oryginalność/wartość - Wartość tekstu polega na zestawieniu wybranych analiz oraz analizie rezultatów polityki ostrego oszczędzania w Niemczech.

\section{Principles and effects of austerity policy in Germany}

KEYWORDS acute saving policy, debt

ABSTRACT Research background - Savings policy in Germany continues to be very popular, although several studies and comparisons have shown that it can have lasting negative effects on economic growth and on the investment level.

Purpose - Explanation of the concept of austerity, analysis of the impact of the implementation of austerity policy in Germany as well as in some other countries, study of the current state of the implementation of the savings policy in Germany.

Research methodology - The study is based on comparative analysis of selected studies and impact analyzes of the policy of saving on growth and on the calculation of own correlation between economic growth and debt.

Findings - The results of the analysis are ambiguous. There are many supporters and opponents of the acute savings policy. Recent analyzes supported by the interpretation of macroeconomic and financial data confirm both, positive and negative effects of the policy of acute savings.

Originality/value - The value of the text is based on the compilation of selected analyzes and analysis of the results of the austerity policy in Germany. 


\section{Einführung}

Die Sparpolitik in Deutschland erfreut sich weiterhin großer Popularität, obwohl mittlerweile nachgewiesen wurde, dass sie dauerhaft negative Effekte auf das Wirtschaftswachstum und auf das Investitionsniveau haben kann.

Das Ziel der vorliegenden Ausarbeitung ist es aufzuzeigen, dass die Sparpolitik, neuerdings auch als Austeritätspolitik bezeichnet, in Deutschland verglichen mit anderen Ländern keine gravierenden negativen Auswirkungen auf das mögliche Wachstum und die wirtschaftliche Leistung hat. Hierzu wurden ausgewählte makroökonomische Studien sowie Daten verglichen und analysiert. Es wurden folgende Hypothesen $(\mathrm{H})$ formuliert:

H1 Konsequent umgesetzte Sparpolitik (Austeritätspolitik) führt in Deutschland zur Haushaltskonsolidierung und zum Abbau der Schulden.

H2 Sparpolitik entlastet zwar den Haushalt, aber sie führt dauerhaft zur Verringerung öffentlicher Ausgaben und Investitionen und hat zusätzlich einen negativen Einfluss auf das Wirtschaftswachstum.

Der Text besteht aus drei Teilen: Im 1. Teil werden die gesetzlichen Grundlagen deutscher Sparpolitik vorgestellt, im Teil 2. wird näher auf den Begriff Austerität eingegangen und es werden einige ausgewählte Studien über die Auswirkungen der Umsetzung der Austeritätspolitik in Deutschland sowie in einigen EU-Ländern erläutert. Im 3. Teil wird der aktuelle Stand der Umsetzung der Sparpolitik in Deutschland beschrieben und untersucht, in welchen Bereichen ggf. negative Effekte auf Grund Sparmaßnahmen sichtbar sind.

\section{Sparpolitik als Teil der Finanzpolitik des Bundes und der Länder}

In der deutschen Geschichte hat die Sparpolitik bzw. das Streben nach Begrenzung von Staatsschulden eine lange Tradition. Beginnend mit dem Preußischen Staatsschuldengesetz aus dem Jahr 1820, haben solche Regelungen auch die späteren Verfassungen (Reichsverfassung von 1871 und die Verfassung der Weimarer Republik von 1919) inkl. das Bundesdeutsche Grundgesetz von 1949 enthalten (Richter, 1869; Wünsche 2015, S. 297 ff.). Das Grundgesetz enthält ebenfalls Regelungen zu Beschränkungen der Möglichkeiten Staatsausgaben über Schulden zu finanzieren. Sollten ggf. neue Schulden aufgenommen werden, ist der Zweck zu prüfen bzw. der außerordentliche Bedarf festzustellen.

Im Zuge späterer Anpassungen des Grundgesetzes, auch in Verbindung mit EU-Vorgaben, sind aktuell vor allem zwei Artikel für die Ausgestaltung der Finanzpolitik maßgeblich:

Art 109 (2) Bund und Länder erfüllen gemeinsam die Verpflichtungen der Bundesrepublik Deutschland aus Rechtsakten der Europäischen Gemeinschaft auf Grund des Artikels 104 des Vertrags zur Gründung der Europäischen Gemeinschaft zur Einhaltung der Haushaltsdisziplin und tragen in diesem Rahmen den Erfordernissen des gesamtwirtschaftlichen Gleichgewichts Rechnung.

(3) Die Haushalte von Bund und Ländern sind grundsätzlich ohne Einnahmen aus Krediten auszugleichen. Bund und Länder können Regelungen zur im Auf- und Abschwung symmetrischen Berücksichtigung der Auswirkungen einer von der Normallage abweichenden konjunkturellen Entwicklung sowie eine Ausnahmeregelung für Naturkatastrophen oder außergewöhnliche Notsituationen, die sich der Kontrolle des Staates entziehen und die staatliche Finanzlage beeinträchtigen, vorsehen. 
und Artikel 115

„(2) Einnahmen und Ausgaben sind grundsätzlich ohne Einnahmen aus Krediten auszugleichen. Diesem Grundsatz ist entsprochen, wenn die Einnahmen aus Krediten 0,35 vom Hundert im Verhältnis zum nominalen Bruttoinlandsprodukt nicht überschreiten.“ (Grundgesetz der BRD, 2009).

Aus den o.g. sowie den weiteren Regelungen des GG in den Art 104a bis 115 leiten sich direkt oder indirekt die Vorgaben und die wichtigsten Ziele der Finanzpolitik ab, die im Weiteren mit der Sparpolitik gleichgesetzt wird:

1. Haushaltsdisziplin und Gleichgewicht innerhalb der Staatsfinanzen sowie die

2. Vermeidung der Aufnahme von Krediten (inkl. sog. Schuldenbremse, die seit 2016 gilt).

Ausgehend von den o.g. Vorgaben ist die Nettokreditaufnahme (dargestellt in der Abb. 1 seit 2014) gesetzeskonform und soll weiterhin fortgesetzt werden. Im Jahr 2016 betrugen die Ausgaben des Bundes laut Finanzplan ca. 312 Mrd. Euro, die Einnahmen ebenfalls ca. 312 Mrd. Euro - somit wird es lt. derzeitiger Planung analog zum Jahr 2016 in den folgenden Jahren keine Neuverschuldung im Bundeshaushalt geben (Finanzplan des Bundes 2015 bis 2019, 2015, S. 8).

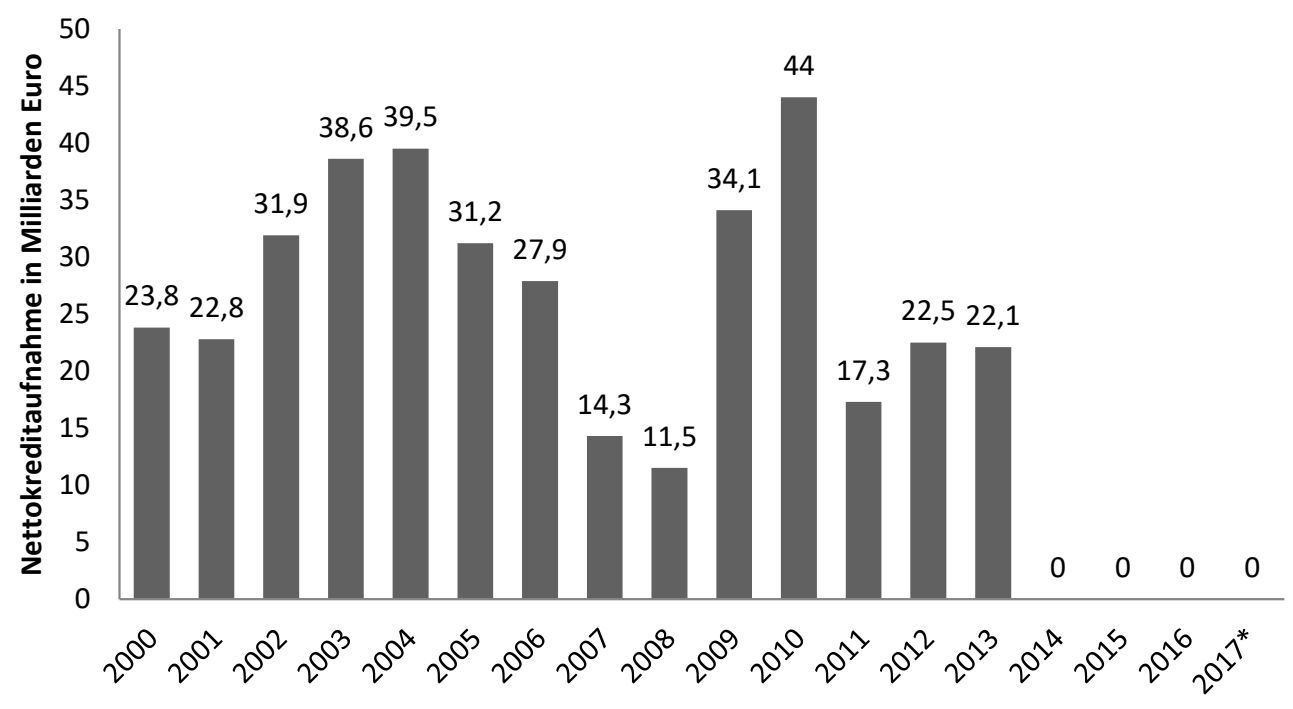

Abbildung 1. Nettokreditaufnahme des Bundes in Mrd. Euro

Quelle: Bundesministerium für Finanzen, http://www.bundesfinanzministerium.de/(1.04.2017).

Bezogen auf die EU-Konvergenzkriterien, die u.a. vorsehen, dass das jährliche Haushaltsdefizit 3\% des BIP nicht überschreiten darf, ist festzustellen, dass Deutschland dieses Kriterium nach dem Jahr 2000 längere Zeit nicht erfüllt hat. Die Überschreitung ist zuletzt auf die Finanz- und Wirtschaftskrise der Jahre 2008-2009 zurückzuführen. Das Haushaltsdefizit ist erst nach mehreren Jahren (1993, 1995-1996, 2001-2005, 2009-2010) unter die geforderte 3\%-Grenze gesunken (siehe Abb. 2). Dieser Umstand ist nicht nur auf die Sparmaßnahmen zurückzuführen, sondern auf das niedrige Zinsniveau in der Eurozone, so gelang die Haushaltskonsolidierung schneller. 


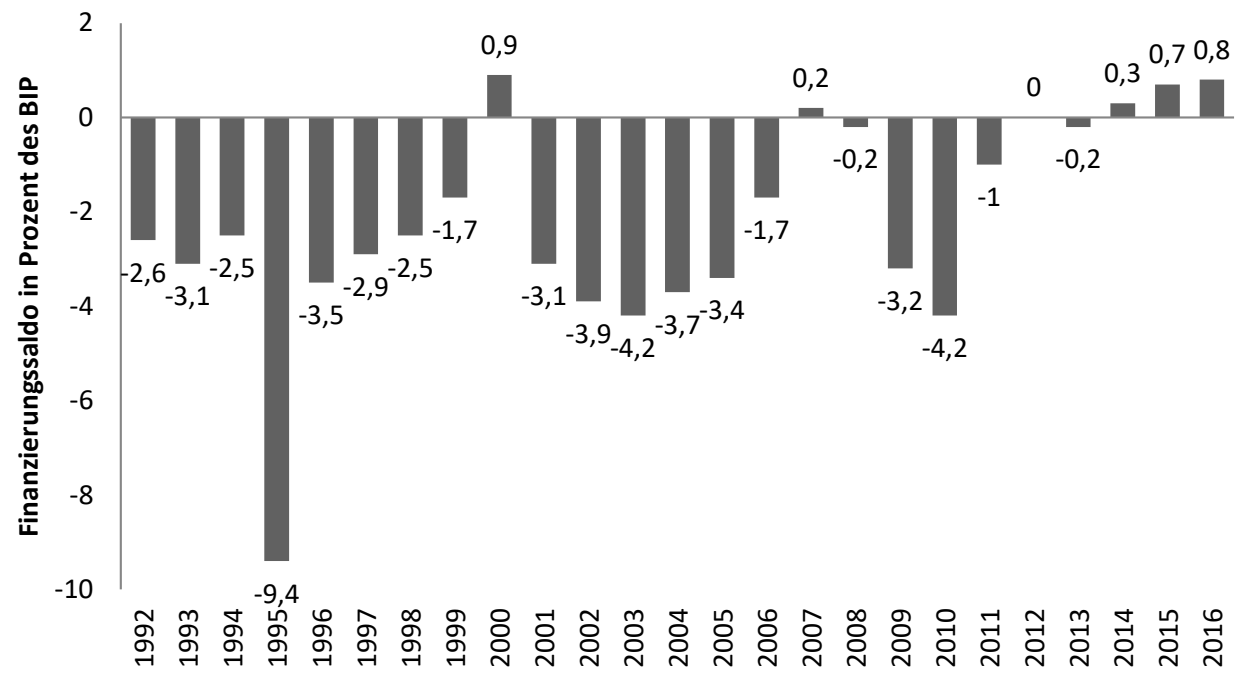

Abbildung 2. Haushaltsdefizit in Deutschland 1992-2016 in \% des BIP

Quelle: Bundesministerium für Finanzen, http://www.bundesfinanzministerium.de/ (1.04.2017).

\section{Von der Sparpolitik zur Austeritätspolitik}

Austerität bezeichnet generelle Kürzungen in den Staatsausgaben und ist als Begriff relativ neu; es beschreibt bzw. wird synonym für Sparpolitik verwendet. Ursprünglich leitet es sich vom altgriechischen ,austēros“ ab und wird als streng oder enthaltsam übersetzt. In der deutschen bzw. auch in der europäischen Geschichte hat Austerität ethische Grundlagen und eine lange Tradition (siehe Tab. 1).

Tabelle 1. Grundlagen der Austerität in europäischer und deutscher Tradition

\begin{tabular}{|l|l|}
\hline \multicolumn{1}{|c|}{ Ethische Grundlagen } & \multicolumn{1}{c|}{ Ökomische Grundlagen } \\
\hline Enthaltsamkeit und Moral als Tugend & Wird als wirtschaftlich effiziente Nutzung angesehen \\
\hline $\begin{array}{l}\text { Begrenzung des Konsums (begründet und vermittelt in der } \\
\text { europäischen Aufklärung) }\end{array}$ & \multirow{2}{*}{$\begin{array}{l}\text { Durch die Änderungen des Konsumverhaltens entstanden } \\
\text { kollektive Konsumformen, z.B. für neueste Produkte } \\
\text { (Handys oder andere Geräte) }\end{array}$} \\
$\begin{array}{l}\text { Altgriechische Tradition, die auf Aristoteles zurückgeht } \\
\text { und später als Gedankengut aufgegriffen und } \\
\text { weiterentwickelt wurde durch Thomas von Aquin, } \\
\text { Voltaire, J.J. Rousseau }\end{array}$ & \\
\hline $\begin{array}{l}\text { Besonders wichtig für die Herausbildung der Einstellung } \\
\text { zur Sparsamkeit als Tugend war das asketische } \\
\text { Element der, ,aufgeschobenen Befriedigung“(deferred } \\
\text { gratification) in Deutschland geprägt durch Max Weber } \\
\text { im Werk Die protestantische Ethik und der Geist des } \\
\text { Kapitalismus }\end{array}$ & Zuwachs an Hedonismus und Individualismus \\
\hline
\end{tabular}

Quelle: Eigene Darstellung auf der Grundlage Dahrendorf (2009); Schui (2014); Stiglitz (2016). 
Da nach dem 2. Weltkrieg kontinuierlich nicht nur die globale Wirtschaft sondern auch die globalen Schulden wuchsen, ist eine kritische Einstellung den Schulden gegenüber nachvollziehbar und auf europäischer Ebene herrscht überwiegend Konsens darüber, dass Schulden abgebaut werden müssen. 2016 erreichte die globale Verschuldung $225 \%$ des weltweiten BIP, darin sind sowohl öffentliche Haushalte als auch die Verbindlichkeiten des privaten Sektors enthalten, die ca. 2/3 der Verschuldung ausmachen (unberücksichtigt wurde dabei der Finanzsektor).

In der Staatstätigkeit wird und kann die Sparpolitik in erster Linie durch die Begrenzung der Ausgaben und der Kreditaufnahme (Nettoneuverschuldung) realisiert werden, mit dem Ziel Staatsverschuldung zu reduzieren. Dabei wird davon ausgegangen, dass sie nicht nur positive Auswirkungen auf die Wirtschaftsprozesse hat, die negativen Folgen werden jedoch in solchen Fällen in Kauf genommen (siehe Tabelle 2).

Tabelle 2. Mögliche positive und negative Auswirkungen staatlicher Sparpolitik

\begin{tabular}{|l|l|}
\hline \multicolumn{1}{|c|}{ Annahme der positiven Effekte } & \multicolumn{1}{c|}{ Kritik bzw. mögliche negative Folgen } \\
\hline Soll vor finanziellen Zusammenbrüchen retten & $\begin{array}{l}\text { Soziale Folgen werden vernachlässigt (der private } \\
\text { Konsum nimmt ab; das wird dabei in Kauf genommen) }\end{array}$ \\
\hline Wird als Notlösung in Krisen angewandt & $\begin{array}{l}\text { Die Wirtschaftsleistung der Länder stagniert und erholt } \\
\text { sich langsamer als in den Ländern, die öffentliche } \\
\text { Ausgaben nicht sofort senken }\end{array}$ \\
\hline $\begin{array}{l}\text { Wird als notwendige, unangenehme Maßnahme } \\
\text { wahrgenommen }\end{array}$ & $\begin{array}{l}\text { Unter Umständen verlängert sich die Krise und bremst } \\
\text { die Rückkehr zum Wachstum }\end{array}$ \\
\hline $\begin{array}{l}\text { Soll monetäre Stabilität wiederherstellen oder } \\
\text { gewährleisten }\end{array}$ & $\begin{array}{l}\text { Führt zu Kürzung der Staatsausgaben für Investitionen in } \\
\text { Infrastruktur und Sozialleistungen }\end{array}$ \\
\hline
\end{tabular}

Quelle: Eigene Darstellung auf der Grundlage Schui (2014); Stiglitz (2016); Blankart (2008).

Seit der sog. Griechenlandkrise bzw. -rettung wird eine sehr strenge Spar- bzw. Austeritätpolitik (AP) zunehmend als Wachstumsbremse kritisiert. Mittlerweile hat sogar der IWF seine Empfehlung zur Sparpolitik revidiert; in Deutschland bzw. in der Eurozone wird sie jedoch weiterhin gemäß gemeinschaftlicher Vorgaben umgesetzt (COUNCIL DECISION of 22 June 2012 abrogating Decision 2010/285/EU; COUNCIL DECISION (EU) 2015/1410 of 19 August 2015). Im Laufe der letzten Jahre haben sich internationale Organisationen, darunter der IMF und IWF, sowie Wirtschaftswissenschaftler mit der Beleuchtung und Untersuchung der Auswirkungen der Umsetzung von AP in verschiedenen Ländern beschäftigt und sprachen einige Empfehlungen aus (siehe Tabelle 3).

Tabelle 3. Ergebnisse ausgewählter Studien

\begin{tabular}{|l|l|l|}
\hline \multicolumn{1}{|c|}{$\begin{array}{c}\text { Autor oder } \\
\text { Institution }\end{array}$} & \multicolumn{1}{|c|}{ Hauptempfehlungen bzw. Hauptergebnisse } & \multicolumn{1}{|c|}{$\begin{array}{c}\text { Betrachteter } \\
\text { Zeitraum }\end{array}$} \\
\hline & $\begin{array}{l}\text { - Lockere Politik der EZB unterstützte das Wirtschaftswachstum und } \\
\text { verhinderte eine erneute Rezession }\end{array}$ & $\begin{array}{l}2007-2009 \\
\text { und Roubini }\end{array}$ \\
Roubini, Mihm & - AP verschlimmert ohne zusätzliche Maßnahmen die Krise & 2013 \\
& - Höhere Haushaltsdefizite für EU-Länder sollten für kurze Zeiträume \\
& möglich sein, um strukturelle Reformen durchzuführen & \\
\hline
\end{tabular}




\begin{tabular}{|c|c|c|}
\hline $\begin{array}{l}\text { Autor oder } \\
\text { Institution }\end{array}$ & Hauptempfehlungen bzw. Hauptergebnisse & $\begin{array}{c}\text { Betrachteter } \\
\text { Zeitraum }\end{array}$ \\
\hline Krugmann (2013) & $\begin{array}{l}\text { - Vergleichsstudie über } 33 \text { Länder, Ergebnis: positive Veränderung des realen } \\
\text { Bruttoinlandsprodukts (BIP) dank Erhöhung staatlicher Ausgaben (eindeutige } \\
\text { Empfehlung für die keynesianische Politik in der Rezessionsphase) } \\
\text { - Durch Senkung der Staatsausgaben und Schulden soll Wettbewerb } \\
\text { angekurbelt werden. }\end{array}$ & $2010-2013$ \\
\hline Stiglitz (2016) & $\begin{array}{l}\text { - Hauptziel der AP ist die monetäre Stabilität, sie führt zur Kürzung der } \\
\text { Staatsausgaben (Investitionen in Infrastruktur und Sozialleistungen) } \\
\text { - Wirtschaftswachstum wäre ohne AP höher } \\
\text { - Die Grundlagen für die Währungsunion (der EU) beruhen auf falschen } \\
\text { Annahmen, ,begrenzte fiskalische Defizite und öffentliche Schuldenstände } \\
\text { seien die entscheidenden Voraussetzungen für eine funktionierende Währung“ } \\
\text { - Einheitliche Regelungen innerhalb der EU bezogen auf Steuersätze wären } \\
\text { sinnvoll; Industriepolitik müsste den neueren EU-Mitgliedsländern ermöglichen } \\
\text { und erlauben den Rückstand aufzuholen }\end{array}$ & $2008-2015$ \\
\hline Heimberger (2016) & $\begin{array}{l}\text { - Länder der Euro-Zone, die stärkere Haushaltskonsolidierungen durchgeführt } \\
\text { haben, hatten einen stärkeren Rückgang des realen BIP (ein Anstieg von } \\
1 \text { Prozentpunkt in der Haushaltskonsolidierung 2011-13 war mit einem } \\
\text { kumulativen Rückgang des realen BIP im gleichen Zeitraum von etwa 1,8\% } \\
\text { verbunden) }\end{array}$ & $2011-2013$ \\
\hline $\begin{array}{l}\text { Holland, Portes } \\
\text { (2012) }\end{array}$ & $\begin{array}{l}\text { - In den Zeiten ohne Krisen neigen die europäischen Länder zu } \\
\text { Haushaltskonsolidierung und erreichen so einen Rückgang der Verschuldung } \\
\text { - Bei niedrigem Wirtschaftswachstum (betrachtet wurde insbesondere } \\
\text { Großbritannien und die Eurozone) erhöht sich der Wert der fiskalischen } \\
\text { Multiplikatoren, sodass Konsolidierung des Haushalts größere Auswirkungen } \\
\text { auf das Wirtschaftswachstum haben } \\
\text { - Zusätzlich treten noch Spillover-Effekte auf, weil die anderen } \\
\text { Volkswirtschaften ähnliche Sparmaßnahmen ergreifen }\end{array}$ & $2011-2013$ \\
\hline $\begin{array}{l}\text { The Good, the Bad, } \\
\text { and the Ugly: } 100 \\
\text { Years of Dealing } \\
\text { with Public Debt } \\
\text { Overhangs } \\
\text { (IFM, 2012) }\end{array}$ & $\begin{array}{l}\text { - Sparpolitik als Instrument sowie die Finanzkonsolidierungsbemühungen } \\
\text { müssten um geeignete Maßnahmen ergänzt werden, damit sie wirksam den } \\
\text { Schuldenabbau unterstützen (inkl. strukturelle und monetäre Bedingungen) }\end{array}$ & $\begin{array}{l}\text { Historische } \\
\text { Daten über } \\
\text { mehrere Jahre } \\
\text { und Länder } \\
\text { mit einem } \\
\text { Staatsdefizit } \\
\text { über } 100 \% \text { BIP }\end{array}$ \\
\hline $\begin{array}{l}\text { Obstfeld, Thomsen } \\
\text { (IMF, 2016) }\end{array}$ & $\begin{array}{l}\text { - } 2016 \text { wird AP nicht mehr explizit von Griechenland gefordert. Die } \\
\text { Maßnahmen innerhalb des ESM-Programms wären für den IWF ausreichend } \\
\text { gewesen } \\
\text { - Griechenland hält sich trotzdem eher an die Vorgaben der europäischen } \\
\text { Institutionen, um Ausgaben zu senken }\end{array}$ & 2016 \\
\hline $\begin{array}{l}\text { Busch, Hermann et } \\
\text { al. (2012) }\end{array}$ & $\begin{array}{l}\text { - Analyse der Auswirkungen der Austeritätspolitik in Griechenland, Italien, } \\
\text { Portugal und Spanien - Austeritätspolitik hat lt. der Autoren definitiv einen } \\
\text { negativen Einfluss auf das Lohnniveau und die Sozialleistungen }\end{array}$ & 2009-2012 \\
\hline $\begin{array}{l}\text { OECD-Prognose } \\
(2016)\end{array}$ & $\begin{array}{l}\text { - Die öffentliche Verschuldung ist krisenbedingt nach } 2008 \text { angestiegen, } \\
\text { anschließende Fiskalpolitik hat die Staatsschuldenquote begrenzt } \\
\text { - OECD-Länder, darunter große Volkswirtschaften, könnten gemeinsame } \\
\text { fiskalische Maßnahmen umsetzen und würden so ihre Produktionszuwächse um } \\
\text { 0,2 Prozentpunkte steigern können } \\
\text { - Empfehlung einer expansiven Fiskalpolitik für die meisten OECD-Länder. } \\
\text { Besonders für Deutschland: mehr Kredite sollten aufgenommen werden, um } \\
\text { öffentliche Investitionen zu erhöhen }\end{array}$ & $\begin{array}{l}1998 \text { mit } \\
\text { Ausblick bis } \\
2018\end{array}$ \\
\hline
\end{tabular}

Quelle: Eigene Darstellung auf der Grundlage: Stiglitz (2016); Holland, Portes (2012); Roubini, Mihm (2010); Krugmann (2013); Heimberger (2016); Busch, Hermann et al. (2012); OECD-Wirtschaftsausblick (2016); IMF (2012, 2016). 


\section{Umsetzung der Sparpolitik in Deutschland}

Unbeirrt der Empfehlungen und internationaler Aufforderungen an Deutschland von der Sparpolitik abzusehen, wird sie in Deutschland weiterhin fortgesetzt. Das Bundesministerium für Finanzen lehnt die Erhöhung der Staatsausgaben ab: „Gesteigerte Staatsausgaben in Deutschland können Strukturprobleme in anderen Volkswirtschaften nicht lösen. Übermäßige Lohnsteigerungen wären kontraproduktiv für Importe und Wohlstand in Deutschland und weltweit.“

Die positive Entwicklung deutscher Wirtschaftsleistung innerhalb der Eurozone ist verschiedenen Umständen zu verdanken. Als Hauptursache wird der häufig für seine Überschüsse kritisierte Export angesehen, aber auch die Lohn- und Produktivitätsentwicklung trugen dazu bei. Hierbei hatte Deutschland zum einen nach 2007 einen öffentlichen Schuldenstand von höchstens 81\% BIP (Tab. 4), zum anderen wurden früh Maßnahmen aus den Konjunkturpaketen umgesetzt, die in 7 meist zukunftsgerichtete Aktionsfelder unterteilt wurden: Informationsgesellschaft, Innovation, Forschung und Entwicklung, Basis-Infrastruktur, IKT-Infrastruktur, Bildung, Gesundheit, Energieeffizienz und Klimaschutz ${ }^{1}$.

Tabelle 4. Öffentlicher Schuldenstand in \% des BIP seit 2007 mit Prognose bis 2020

\begin{tabular}{|l|c|c|c|c|c|c|c|c|c|c|c|c|c|c|}
\hline \multicolumn{1}{|c|}{ Land } & 2007 & 2008 & 2009 & 2010 & 2011 & 2012 & 2013 & 2014 & 2015 & 2016 & 2017 & 2018 & 2019 & 2020 \\
\hline Deutschland & 63,5 & 64,9 & 72,4 & 81,0 & 78,3 & 79,5 & 77,1 & 74,5 & 71,0 & 68,2 & 65,9 & 63,6 & 61,1 & 58,9 \\
\hline Griechenland & 103,1 & 109,4 & 126,7 & 146,2 & 172,1 & 159,6 & 177,7 & 180,1 & 176,9 & 183,4 & 184,7 & 184,7 & 178,5 & 173,1 \\
\hline Portugal & 68,4 & 71,7 & 83,6 & 96,2 & 111,4 & 126,2 & 129,0 & 130,2 & 129,0 & 128,4 & 128,2 & 127,7 & 127,0 & 126,5 \\
\hline Eurozone & 64,9 & 68,5 & 78,3 & 84,1 & 86,7 & 91,3 & 93,3 & 94,3 & 92,5 & 91,7 & 91,0 & 89,8 & 88,1 & 86,2 \\
\hline USA & 64,0 & 72,8 & 86,0 & 94,7 & 99,0 & 102,5 & 104,6 & 104,6 & 105,2 & 108,2 & 108,4 & 107,9 & 107,8 & 107,9 \\
\hline
\end{tabular}

Quelle: World Economic and Financial Surveys (2016), S. 69.

Alle umgesetzten Konjunkturpakete bzw.-programme zielten darauf ab, die Auswirkungen der Krise zu mildern und entsprachen in ihrem Wesen der keynesianischen Wirtschaftspolitik, die jedoch in Deutschland mit den Grundlagen der sozialen Marktwirtschaft und dem Ordoliberalismus konkurriert. Daraus ergeben sich weitere wesentliche Unterschiede in der dt. Einstellung zur Sparpolitik und zur Rolle des Staates: „Die wirtschaftliche Tätigkeit des Staates sollte auf die Gestaltung der Ordnungsformen der Wirtschaft gerichtet sein, nicht auf die Lenkung des Wirtschaftsprozesses“ (Eucken, 2005). Damit wird auf die Rahmenbedingungen für funktionierende Märkte Wert gelegt; der Staat soll regulieren, nicht stimulieren (Blyth, S. 90). Dies deckt

1 Konjunkturpakete wurden zu dieser Zeit ebenfalls weltweit umgesetzt, um weiteren negativen Entwicklungen der Finanzmärkte und in der Realwirtschaft entgegenzuwirken. Dafür wurden weltweit ca. 2 Billionen Euro für Wirtschaftsprogramme zur Unterstützung der Volkswirtschaften, zum Schutz und zur Unterstützung von unternehmerischen Aktivitäten sowie für die Erhaltung der Arbeitsplätze zur Verfügung gestellt. Die USA haben weitreichende Programme mit einem Volumen von 733,0 Mrd. € (5,6\% des BIP im Jahr 2008) für die Stabilisierung und Belebung der Wirtschaftsleistung aufgestellt. China hat Unterstützung in einem Umfang von 429,5 Mrd. €, Russland 89 Mrd. $€$, Deutschland 82 Mrd. $€(3 \%$ des BIP im Jahr 2008), Spanien 49,1 Mrd. €, Großbritannien 28,9 Mrd. € (1,4\% des BIP) und Frankreich 28,6 Mrd. € (0,6\% des BIP) gewährt. Die „Anti-Krisen-Pakete“ wurden größtenteils zur Verbesserung der bestehenden, oft vernachlässigten Infrastruktur, eingesetzt und belebten nur kurzfristig die Konjunktur; Investitionen in die Zukunft, Förderung umweltfreundlicher Technologien und deren Entwicklung haben wiederum langfristige Auswirkungen. 
sich mit der unterschiedlichen Auslegung des Nutzens und der Einhaltung der Regeln der Sparpolitik - Stiglitz und Krugmann (vgl. Tab. 3) als Befürworter der keynesianischen Wirtschaftspolitik sehen in den Konjunkturpaketen eine stimulierende Wirkung und vernachlässigen die Kosten. Kurzfristig hat auch Deutschland Mittel aus Konjunkturpaketen zur Verfügung gestellt und bei Betrachtung ihrer Auswirkungen -Wirtschaftswachstum hat wieder eingesetzt- waren sie wirksam (zum Teil gestützt durch die Exportleistung bzw. durch das Wirken der jeweiligen Konjunkturpakete in den Importländern).

Ab 2010 wirkte zudem noch die Entlastung durch das niedrige Zinsniveau in der Eurozone, so gelang in Deutschland der Schuldenabbau und die Haushaltskonsolidierung schneller (Abbildung 3). Innerhalb der EU ist die Einhaltung der Regeln der AP auf Grund unterschiedlicher Mechanismen, die die Auswirkungen der Krise in den einzelnen Ländern vertieft haben, schwierig (Griechenland - Senkung der Ausgaben, hohe Zinsbelastung, Italien - nach 2012 Senkung der Ausgaben und Erhöhung der Steuern, Irland und Spanien - Immobilienblasen). Auf politischer Ebene hören die EU-Länder nicht auf, sich um Nichtigkeiten zu streiten, statt mehr einheitliche (Steuer-)Regeln einzuführen (Pikkety, 2015). Schon 2016 und weiterhin 2017 geht der Trend in der EU, sich nicht auf Gemeinsamkeiten zu berufen, sondern auf Unterschiede und sollte es tatsächlich zum BREXIT kommen, wird es auch negative Auswirkungen auf verbleibende EU-Länder haben. Nichtsdestotrotz ist die Verpflichtung eines jeden EU-Landes unter den festgelegten Defizit- und Schuldengrenzen zu bleiben, unumgänglich und ergibt sich aus dem Anhang zum Vertrag von Maastricht und wird bei Überschreitung geahndet, indem ein Verfahren bei einem übermäßigen Defizit eingeleitet wird (Excessive Deficit Procedure - EDP).

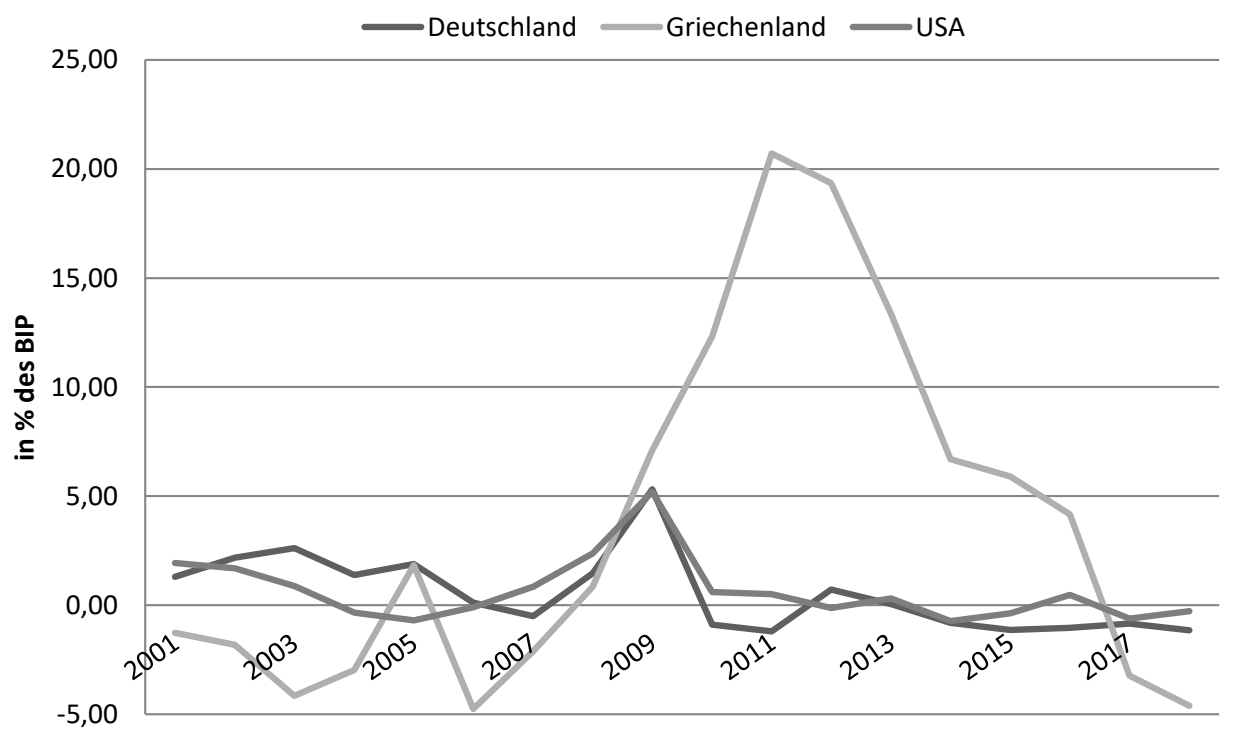

Jahr

Abbildung 3. Konsolidierung der Staatsausgaben in \% des BIP nach dem Verfahren bei einem übermäßigen Defizit

Quelle: AMECO, Snow ball effect on general government consolidated gross debt, https://ec,europa,eu/info/business-economy-euro/indicators-statistics/economic-databases/macro-economic-database-ameco_en (20.04.2017). 
Um Wachstum anzutreiben, wird als Möglichkeit die Erhöhung des Investitionsniveaus empfohlen. Das Investitionsniveau bleibt trotz niedriger Zinsen unter dem Wert früherer Jahre bzw. Dekaden bis auf die Ausnahmejahre, in denen Investitionen aus den Konjunkturpaketen getätigt und gefördert wurden (European Economic Forecast Winter, 2017, S. 11). Auch für Deutschland kann man dabei keinen positiven Trend feststellen. Eine Kausalbeziehung zwischen den Bruttoanlageinvestitionen des Staates in \% des BIP und der Veränderung des Schuldenstandes des Staates mittels Korrelation nach Pearson konnte nach 2006 keinen Zusammenhang für Deutschland nachweisen (der Wert von $r=-0,6$ deutet auf eine schwache Korrelation hin).

Die Analyse der Ausgaben des Bundes zeigt auf, dass der Bereich „Arbeit und Soziales“ durch das ausgebaute Transfersystem fast 40\% der Ausgaben ausmacht. Die Infrastrukturausgaben beliefen sich nach 2011 auf ca. 8\% der Ausgaben. Es gab eine geringfügige Verlagerung der Schwerpunkte. Erst ab 2016 wurden die Kommunen wieder in einigen Bereichen entlastet, u.a. bei der Bewältigung steigenden (Sozial-)Ausgaben, bei der Steigerung der Investitionstätigkeit durch die Aufstockung des Kommunalinvestitionsförderungsfonds um 3,5 Mrd. Euro sowie bei der Aufnahme, Unterbringung und Integration von Flüchtlingen (Monatsbericht des BMF, Januar 2017, S. 23).

Tabelle 5. Verteilung der geplanten Ausgaben des Bundes nach Sektoren laut Finanzplan von 2011 bis 2017 in $\%$

\begin{tabular}{|l|c|c|c|c|c|c|c|}
\hline Sektor & 2011 & 2012 & 2013 & 2014 & 2015 & 2016 & 2017 \\
\hline Arbeit und Soziales & 42,90 & 41,4 & 39,30 & 40,90 & 41,70 & 40,80 & 42,20 \\
\hline Verteidigung & 10,30 & 10,40 & 11,00 & 11,10 & 10,80 & 11,00 & 11,10 \\
\hline $\begin{array}{l}\text { Verkehr und digitale } \\
\text { Infrastruktur }\end{array}$ & 8,10 & 8,30 & 8,50 & 8,60 & 7,70 & 7,80 & 8,20 \\
\hline Bundesschuld & 12,40 & 13,10 & 11,00 & 10,30 & 9,40 & 8,00 & 6,10 \\
\hline Bildung und Forschung & 3,80 & 4,20 & 4,60 & 4,70 & 5,10 & 5,30 & 5,30 \\
\hline Gesundheit & 5,10 & 4,70 & 4,10 & 3,80 & 4,00 & 4,70 & 4,60 \\
\hline $\begin{array}{l}\text { Allgemeine } \\
\text { Finanzverwaltung }\end{array}$ & 3,80 & 4,10 & 6,80 & 5,40 & 4,70 & 4,90 & 4,50 \\
\hline $\begin{array}{l}\text { Familie, Senioren, Frauen } \\
\text { und Jugend }\end{array}$ & 2,10 & 2,10 & 2,40 & 2,60 & 2,80 & 2,90 & 2,80 \\
\hline Inneres & - & - & - & - & - & - & 2,50 \\
\hline $\begin{array}{l}\text { Wirtschaftliche } \\
\text { Zusammenarbeit }\end{array}$ & - & - & - & 2,10 & 2,20 & 2,40 & 2,40 \\
\hline Wirtschaft und Energie & 2,00 & 2,00 & 2,00 & - & 2,40 & 2,40 & 2,30 \\
\hline Sonstige & 9,50 & 9,80 & 10,30 & 10,50 & 9,20 & 9,80 & 8,00 \\
\hline Summe & 100,00 & 100,00 & 100,00 & 100,00 & 100,00 & 100,00 & 100,00 \\
\hline
\end{tabular}

Quelle: Finanzplan des Bundes 2016 bis 2020. (2016), S. 15.

Erst bei einer langfristigen Betrachtung der Daten - seit 1970 - sind zwei Trends zu beobachten: ein steter Rückgang der Investitionsausgaben und gleichzeitig ein Anstieg der Sozialausgaben. Es ist allerdings nicht eindeutig, ob exogene oder endogene Faktoren die Verschuldung antreiben, bei den endogenen Faktoren könnte der politische Einfluss eine Rolle spielen 
(Holtfrerich, 2015). Gleichzeitig ist nicht ganz eindeutig, ob der Policy-Mix oder lediglich das Ausbleiben von höheren Wachstumsraten den Schuldenstand erhöht haben.

Nicht nur die OECD, sondern auch deutsche Wirtschaftswissenschaftler, u.a. Bofinger und Fratscher empfehlen den Einsatz der gesparten Mittel für öffentliche Investitionen, insbesondere für die Infrastruktur, um dem Wertverlust des öffentlichen Kapitalstocks entgegenzuwirken. ${ }^{2}$

Bei der Betrachtung der Wachstumsraten in der Abbildung 4 kann man für Deutschland nicht feststellen, dass die Sparpolitik eine signifikante negative Wirkung auf das Wirtschaftswachstum hatte. Die Wachstumsraten in Deutschland liegen in den letzten Jahren weit unter dem Niveau 1960er oder 1970er Jahre. Die deutsche Wirtschaft hat sich nach der letzten weltweiten Finanz- und Wirtschaftskrise 2009 relativ schnell erholt und weist schon seit 2010 positives Wachstum auf.

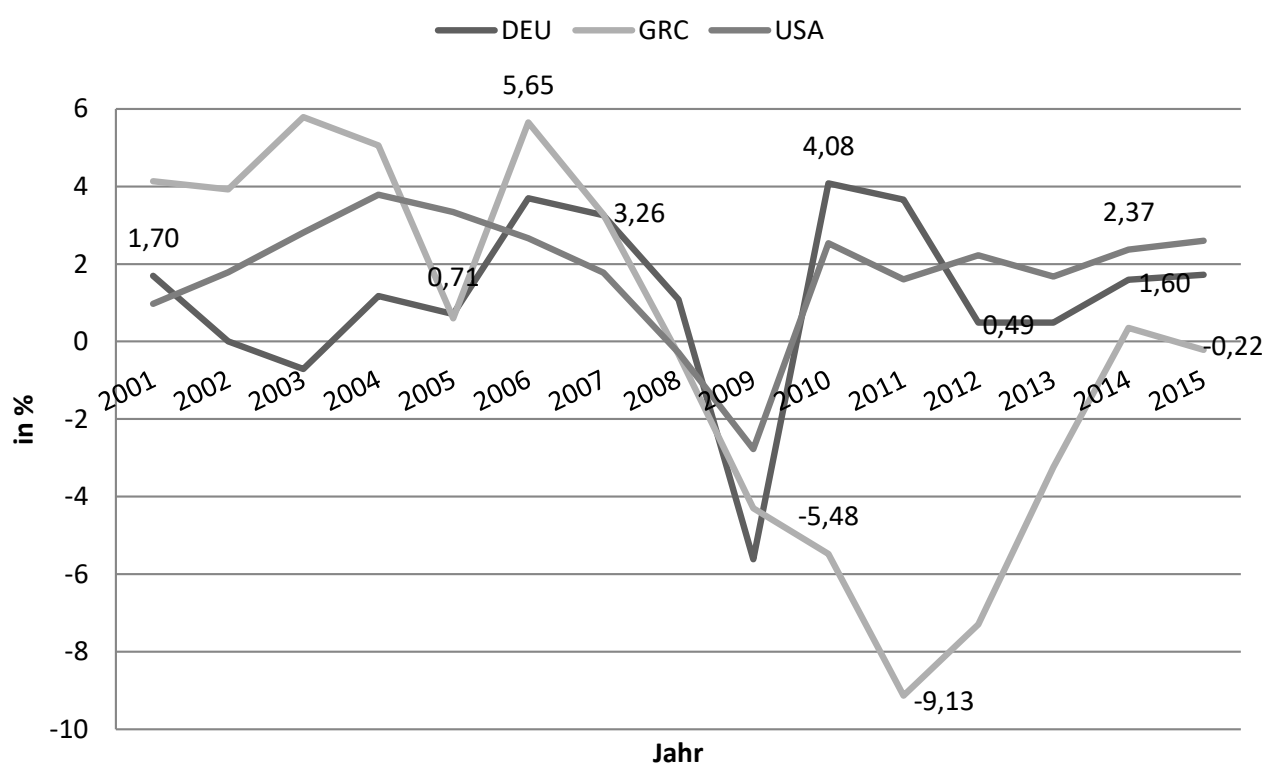

Abbildung 4. Wirtschaftswachstum in \%

Quelle: World Bank, http://databank.worldbank.org/data/reports.aspx?source=2\&series=NY.GDP.-MKTP.KD.ZG\&country=\#n (20.04.2017).

\section{Fazit}

Die aufgeführten Beispiele (Ergebnisse der Studien und Analysen) sind ambivalent. Es gibt zahlreiche Befürworter und Gegner der Austeritätspolitik (AP), die jeweils ihre Meinung durch entsprechende Auslegung der Makro- und Finanzdaten der einzelnen Länder belegen.

2 Der Wertverlust wurde an Hand des Autobahnnetzes errechnet: Insgesamt besitzt Deutschland $12.950 \mathrm{~km}$ Autobahnen (2015), die in ihrem gegenwärtigen Zustand ca. 82 Mrd. Euro wert sind (6,3 Mio. pro km). Der Wert der Autobahnen ist seit 1991 mit 69\% des Neupreises auf 58\% 2015 kontinuierlich gesunken. Vgl. Die Staatsschulden sind gedeckt (2017), S. 25. 
Zusammenfassend lässt sich feststellen, dass die AP in manchen Fällen positive Auswirkungen haben kann, sie muss jedoch durch unterschiedliche Maßnahmen begleitet werden und soll kein Dauerzustand sein. Es gibt Faktoren, die in den Phasen, in denen ein Land AP umsetzt, positiv die Wirtschaftslage beeinflussen können. Dazu können begleitende Maßnahmen z.B. aus steuerlichen oder fiskalischen Anreizen bestehen, um das Wachstum oder auch die Struktur der Produktion und des Exportes zu fördern. Das letzte sichert Deutschland eine starke Position innerhalb der europäischen Volkswirtschaften, obwohl die Wirtschaftssubjekte nur kurzfristig mittels Konjunkturpakete unterstützt wurden.

Die ausgewählten Beispiele zeigen weiterhin, dass Maßnahmen, die in einer Volkswirtschaft positive Auswirkungen haben wie die AP in Deutschland in anderen nicht zu gleichen Ergebnissen führen. Die Hypothese 1 konnte für Deutschland verifiziert werden (die konsequent umgesetzte Sparpolitik (Austeritätspolitik) führt in Deutschland zur Haushaltskonsolidierung und zum Abbau der Schulden).

Die Ergebnisse der Überprüfung der 2. Hypothese sind nicht eindeutig: Die Sparpolitik hat den Haushalt in Deutschland entlastet; die Verringerung der öffentlichen Ausgaben kann aber zum Teil auf das niedrige Zinsniveau zurückgeführt werden, nicht auf die AP selbst. Die Ausgaben für Investitionen sind seit Jahren auf einem eher konstanten niedrigen Niveau und werden erst ab 2017 erhöht. Ein signifikanter negativer Einfluss aus das Wirtschaftswachstum konnte auf Grund der AP nicht nachgewiesen werden; es sei allerdings anzunehmen, dass die Wachstumsrate ohne Sparmaßnahmen hätte höher ausfallen können. In diesem Bereich sind weiterführende Analysen empfehlenswert.

\section{Literatur}

Blankart, Ch. (2008). Öffentliche Finanzen in der Demokratie. München: Vahlen Verlag.

Blyth, M. (2014). Wie Europa sich kaputt spart. Bonn: Dietz Verlag.

Busch, K., Hermann, Ch. et al. (2012). Eurokrise, Austeritätspolitik und das Europäische Sozialmodell. Wie die Krisenpolitik in Südeuropa die soziale Dimension der EU bedroht. Berlin: Friedrich-Ebert-Stiftung.

Dahrendorf, R. (2009). Vom Sparkapitalismus zum Pumpkapitalismus. Cicero, 8.

Die Staatsschulden sind gedeckt (2017). Handelsblatt Nr. 24.

Eucken, W. (2005). Grundsätze der Wirtschaftspolitik. Poznań: Wyd. Poznańskie.

Finanzplan des Bundes 2015 bis 2019 (2015). Drucksache 18/5501, 18. Wahlperiode, 14.08.2015.

Grundgesetz der Bundesrepublik Deutschland (2009). Berlin: Deutscher Bundestag.

Heimberger, Ph. (2016). Did Fiscal Consolidation Cause the Double-Dip Recession in the Euro Area? WIIW, Oktober 2016.

Holland, D., Portes, J. (2012). Self-defeating austerity? National Institute Economic Review, 222.

Holtfrerich, C.L., Feld, L., Heun, W. et al. (2015). Staatsschulden: Ursachen, Wirkungen und Grenzen. Berlin: Union der deutschen Akademien der Wissenschaften e. V.

Monatsbericht des BMF, Januar 2017 (2017). Berlin.

Monatsbericht des BMF, März 2017 (2017). Berlin.

OECD-Wirtschaftsausblick (2016), Ausgabe 2016/2. Paris: OECD Publishing.

Piketty, Th. (2015). Die Schlacht um den Euro. Interventionen. München: Verlag C.H. Beck.

Richter, E. (1869). Das Preußische Staatsschuldenwesen und die Preußischen Staatspapiere. Berlin: Maruschke und Behrend.

Roubini, N., Mihm, S. (2010). Das Ende der Weltwirtschaft und ihre Zukunft. Frankfurt/M: Campus Verlag. 
Schui, F., Austerität. (2014). Politik der Sparsamkeit: Die kurze Geschichte eines großen Fehlers. München: Karl Blessing Verlag.

Stiglitz, J. (2016). Europa spart sich kaputt. Warum die Krisenpolitik gescheitert ist und der Euro einen Neustart braucht. München: Siedlerverlag.

The Good, the Bad, and the Ugly: 100 Years of Dealing with Public Debt Overhangs (2012). World Economic and Financial Surveys, World Economic Outlook October 2012, International Monetary Fund (IMF).

World Economic and Financial Surveys, Fiscal Monitor, Debt Use It Wisely (2016). Washington: IMF.

Wünsche, H.F. (2015). Ludwig Erhards Soziale Marktwirtschaft. München: Lau Verlag.

\section{Internetquellen}

AMECO, Snow ball effect on general government consolidated gross debt, https://ec,europa,eu/info/business-economy-euro/indicators-statistics/economic-databases/macro-economic-database-ameco_en.

BESCHLÜSSE COUNCIL DECISION (EU) 2015/1410 of 19 August 2015 giving notice to Greece to take measures for the deficit reduction judged necessary to remedy the situation of excessive deficit, Amtsblatt der Europäischen Union 20.8.2015. Heruntergeladen von: http://eur-lex.europa.eu/legal-content/DE/TXT/PDF/?uri=CELEX: 32015D1410\&from $=\mathrm{EN}$.

Bundesministerium für Finanzen. Heruntergeladen von: http://www.bundesfinanzministerium.de/

Blog des IMF: The IMF is Not Asking Greece for More Austerity, Posted on December 12, 2016 by iMFdirect, Maurice Obstfeld and Poul M. Thomsen. Heruntergeladen von: https://blog-imfdirect.imf.org/2016/12/12/the-imf-is-not-asking-greece-for-more-austerity/.

Blog von P. Krugmann. Heruntergeladen von: https://krugman.blogs.nytimes.com/2015/01/06/the-record-of-austerity/? module $=$ BlogPost-Title \&version $=\mathrm{B} \log \% 20$ Main\&contentCollection $=$ Opinion \&action $=$ Click \&pgtype $=$ Blogs\&region=Body\&_r=1.

COUNCIL DECISION of 22 June 2012 abrogating Decision 2010/285/EU on the existence of an excessive deficit in Germany (2012/369/EU), Official Journal of the European Union. Heruntergeladen von: http://ec.europa.eu/economy_finance/economic_governance/sgp/pdf/30_edps/126-12_council/2012-06-22_de_126-12_council_en.pdf

European Economic Forecast Winter 2017 (2017). EU 2017, ISSN 2443-8014 (online).

\section{Cytowanie}

Jodkowska, L. (2017). Grundsätze und Auswirkungen der Sparpolitik in Deutschland. Wspótczesne Finanse. Teoria i Praktyka, 1 (2), 13-24. DOI: 10.18276/wf.2017.1-02. 